ABSTRACTS. PHYTOPHARM 2012

Characteristics of the obtained hydrolisates

\begin{tabular}{|c|c|c|c|c|c|c|}
\hline \multirow{2}{*}{ Sample } & \multicolumn{2}{|c|}{$A A, \%$} & \multicolumn{2}{|c|}{$\mathrm{P}, \%$} & \multicolumn{2}{|c|}{ DPPH: $\mathrm{IC}_{50}, \mathrm{mg} / \mathrm{ml}$} \\
\hline & $1.5 \mathrm{~h}$ & $3 \mathrm{~h}$ & $1.5 \mathrm{~h}$ & $3 \mathrm{~h}$ & $1.5 \mathrm{~h}$ & $3 \mathrm{~h}$ \\
\hline Native CF* & $3.5 \pm 0.3$ & $4.4 \pm 0.3$ & $9.5 \pm 0.3$ & $10.2 \pm 0.4$ & $8.2 \pm 0.2$ & $8.5 \pm 0.3$ \\
\hline A & $4.5 \pm 0.2$ & $8.2 \pm 0.4$ & $13.9 \pm 0.3$ & $19.3 \pm 0.4$ & $5.5 \pm 0.3$ & $5.0 \pm 0.3$ \\
\hline $\mathrm{F}$ & $11.0 \pm 0.4$ & $12.5 \pm 0.5$ & $15.7 \pm 0.4$ & $22.4 \pm 0.5$ & $4.4 \pm 0.2$ & $3.5 \pm 0.2$ \\
\hline P6L & $4.5 \pm 0.2$ & $7.2 \pm 0.3$ & $12.0 \pm 0.4$ & $15.5 \pm 0.3$ & $7.1 \pm 0.3$ & $5.9 \pm 0.4$ \\
\hline
\end{tabular}

DPPH free radical scavengers: $I C_{50}=3.5 \pm 0.2 \mathrm{mg} / \mathrm{ml}$ in comparison with native $\mathrm{CF}$ and other $\mathrm{CFHs}$.

References: (1) H.-C.Wu et al. (2003) Food Res. Intern., 36: 949-957. (2) F. C. Church et al.
(1983) J. Dairy Sci., 66: 1219-1227. (3) O. Warburg et al. (1942) Biochem. Z., 310: 384-421. (4) N. Rosoiu et al. (2008) Rom. Soc. Biol. Sci. 2008, 13 (5): 3944-3954.

\title{
PRELIMINAR ANALYSIS OF THE COMPOSITION AND ACTION MECHANISM OF THE COMPLEX PREPARATION OF CODFISH LIVER
}

\section{() Maltseva A. L., Urakova I. N., Pozharitskaya O. N., Makarov V. G.}

\section{St-Petersburg Institute of Pharmacy, 47/5, Piskarevsky prosp., 195067, St-Peters-burg, Russia}

The preparation includes diverse biological substances obtained from multistep processing of codfish liver. Previous studies demonstrated strong anti-inflammatory activity in contact dermatitis model (1). The purpose of this work was to characterize in outline composition and possible action mechanism of the preparation. Primary fractionation on molecular weight (MW) and estimation of effect on functional activity of professional phagocytes (on their capability to produce reactive oxygen species, ROS) were carried out. Lipophilic fraction was separated from aqueous preparation suspension by centrifugation; other fractions were obtained by sequential ultrafiltrational exclusion of components with
MW exceeding $10 \mathrm{kDa}$ and $1 \mathrm{kDa}$. The ratio of fractions (fig.1) demonstrates the prevalent presence (54\%) of low-MW components, which showed no reliable effect on ROS production (fig.2).

The strongest effect was detected in lipophilic fraction («lipids», fig. 2) and this effect four times exceeds one of total preparation (at the same concentration) and of $0,01 \%$ zymosan suspension. The exact relation between stimulatory effect on phagocytes and general anti-inflammatory action of the preparation require further investigations.

Reference: (1) K. L. Kryshen et al. (2012) Exp. Clinic. Dermatocos. 2: 38-42.
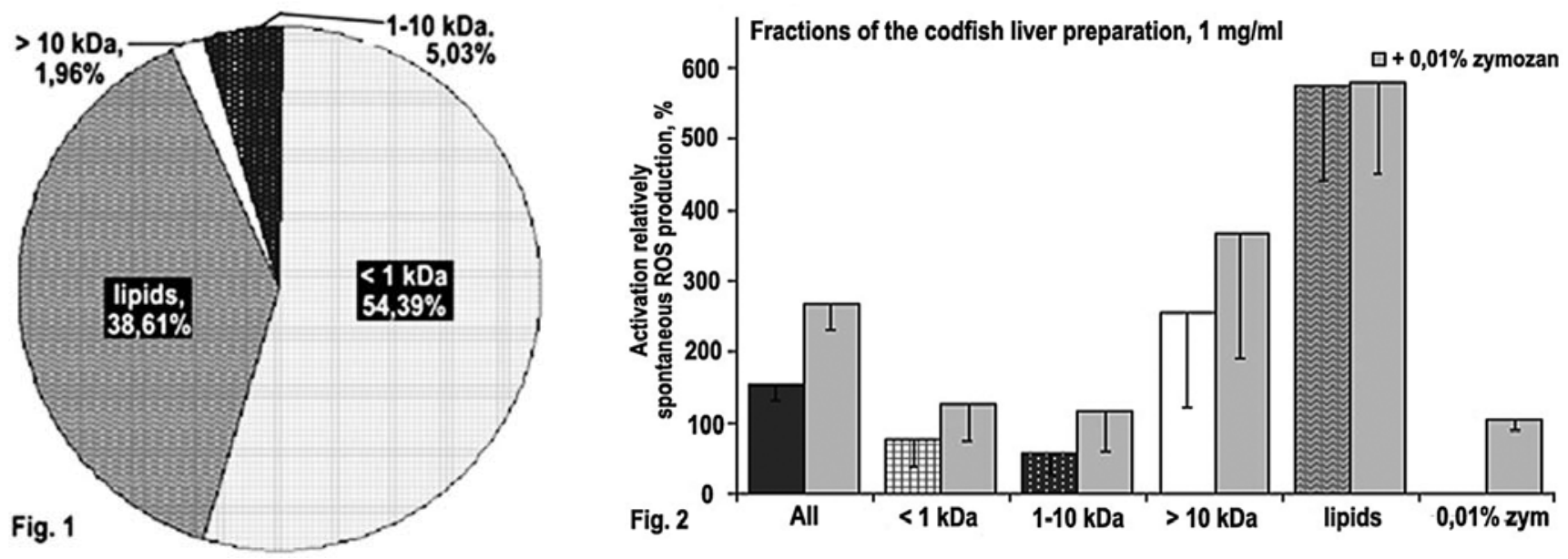